\title{
Smart Health Records
}

\author{
Veronika Nugraheni Sri Lestari, Robbi Rahim, Phong Thanh Nguyen, Wahidah Hashim, Andino \\ Maseleno
}

\begin{abstract}
If there is no health record history of a patient then it will be very difficult for treating in different hospitals. Especially for travelers and in the case of emergency it can ruin the auspicious treatment and diagnosis. If the smart health records are based on Cloud then there is higher difficulties for protection and security, the open difficulties of interoperability and joining and also lack of every day support for the high accessibility of wellbeing history. The smart health records that are already exists can store only limited about of data of patients and they stored data only for specific hospital and do not support versatility of patients crosswise over various medical clinics. The technology that use better treatment for patients, improve quality of life for everyone and provide better diagnostic tools can be consider as smart health.
\end{abstract}

Keywords : smart health; records; cloud; hospitals.

\section{INTRODUCTION}

The healthcare services that are provide the services for treatment, diagnosis, and prevention form diseases and controlled by the good management team can be consider as smart health care [1]. By connecting healthcare with information technology this type of system provide data for patient at any time or any place. Digital transformation is very necessary in smart health field and it associated with the fourth mechanical upheaval for example intermingling of technology and science, hyper-connectivity, and super intelligence [2,3] in the industry of healthcare. In recent years a technology is emerged with the combination of cloud computing [4], big data technologies [5,6], Internet of Things (IoT) [7], and existing types of data and correspondence innovation (ICT) [8,9]. The example of this type of technology are "mobile health" [m-health] $[8,9]$, tele-health," [12-15] and "u-health" [10,11]. IoT empowers physical gadgets to gather and trade information in a mechanized manner.

Smart health incorporated with a broadly versatile clinical information archive that is emphatically eHealth benchmarks consistent, offers a scope of off-the-rack wandering consideration and the other public and private eHealth systems connected with it. The smart health record

Revised Manuscript Received on July 22, 2019.

* Correspondence Author

Veronika Nugraheni Sri Lestari, Universitas Dr. Soetomo, Surabaya, Indonesia.E-mail: venugra@unitomo.ac.id

Robbi Rahim, Sekolah Tinggi Ilmu Manajemen Sukma, Medan, Indonesia.

Phong Thanh Nguyen*, Department of Project Management, Ho Chi Minh City Open University, Vietnam. E-mail: phong.nt@ou.edu.vn

Wahidah Hashim, Institute of Informatics and Computing Energy, Universiti Tenaga Nasional, Malaysia.

Andino Maseleno, Institute of Informatics and Computing Energy, Universiti Tenaga Nasional, Malaysia. features are as follows:

1. The clinic and EHR Repository solution based on smart health record provide high degree of authentication, privacy, consent management and security.

2. To support the described point of care to the clinical management the smart health provides other health modules and a range of chronic disease data. To support health program data sets and new chronic disease the solution is little costly. To fulfill the new clinical management needs on line forms to get specific data and it can be developed and deployed fast.

3. The other health modules and chronic disease of Smart Health specialist provide described specific data. It connects to the EMR system of hospitals. In health services to ensure that smart health records are available for clinicians Smart Health modules interoperate with EMR systems.

4. There are several cloud based smart health records are available. The example of cloud based Smart Health data is secure in centre located in Australia and it Electronic Health Record Repository operates from an ISO27001. The clinical information system of smart health and repository services provide the data to other information systems and they support the services to national eHealth infrastructure. The smart health records support the health services, administrative system of hospital and patient, diagnostic services, clinical systems and dispensing systems.

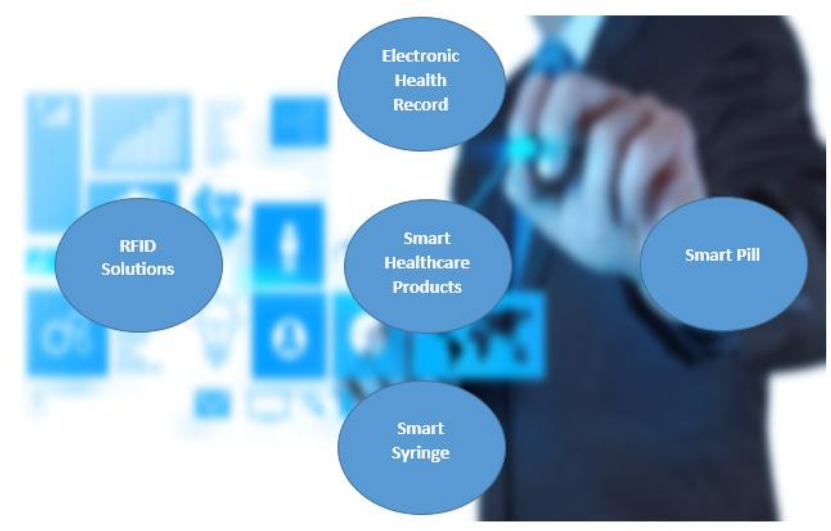

Figure 1: smart healthcare product's components

\section{HISTORY OF HEALTH RECORDS}

In the ancient era the medical history of any patient is recorded on paper. Because it was very simple for using, it was durable and the cost was very low. When the medical professionals caring for a patient the Information may be shared and quickly added. Due to decentralize nature of the healthcare system there were many problems arise.

Published By:

Blue Eyes Intelligence Engineering 


\section{Smart Health Records}

There were very difficult to get the record of patient when it visits different offices treatment like primary care, cardiology, dental work, etc. Incomplete and Fragmented record of patient created problems in proper care of patients. So there is need to develop a electronic based smart health record that can solve the problems related to record that were traditionally saved on papers.

\section{SMART HEALTHCARE}

As previously defined that smart health is provide a digital healthcare solution, which can controlled remotely. Mobile-health and telemedicine and collectively known as smart health. Smart health provides automatic continuous monitoring of health; alter suitable solution and emergency detection. For enhancing the efficiency and quality of healthcare the connected health system enable self-care services and can operate with remote access. It is used in the area of telemedicine, where the user knows about its health and when it required provide the feedback. The system of smart healthcare provide a complete solution of operate autonomously; when it require a feedback solutions from the clinicians it can directly connected to them. For people or hospitals based on cost, architecture and power the healthcare network can be developed.

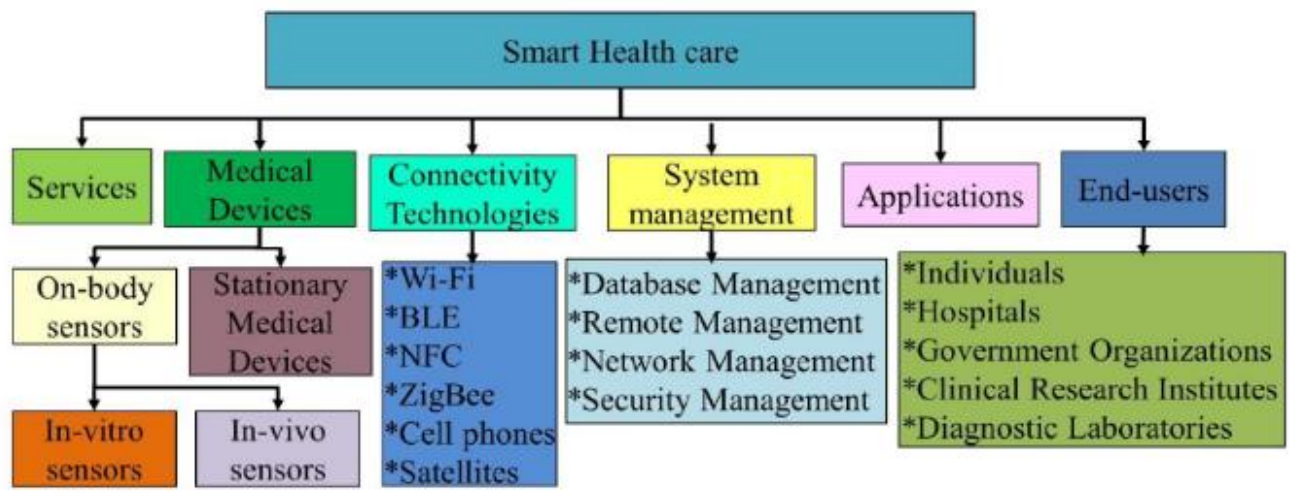

Figure 2: Smart Health Care classification [18]

\section{ARCHITECTURE OF SMART HEALTHCARE}

Functional requirements and non-functional requirements are the two main requirements of smart healthcare system. The specific requirements of a particular smart healthcare architecture are address by Functional requirements. For example based on the application if a temperature monitoring system is developed then the thermistor and thermometer operation, frequency and data collection mechanism can vary. In this way according to the applications how it used in the healthcare system the functional requirements are special to each component.

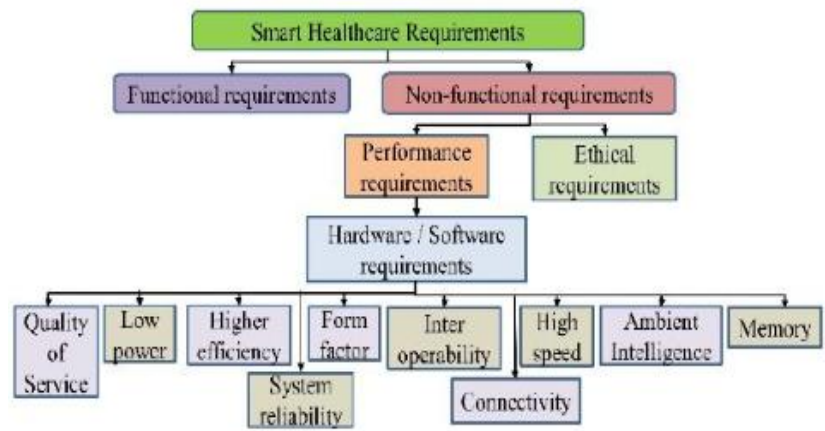

Figure 3: Requirements in Smart Health Care [18]

The performance requirements of complete smart healthcare system can be divided in two parts like software and hardware. The necessary requirements for accurate and efficient smart health care systems are:

1. ability to interoperate across different platforms

2. ease of deployment

3. continuous support of technology
4. enriched user experience

5. small form factor

6. quality of service

7. low power

8. easily upgradeable

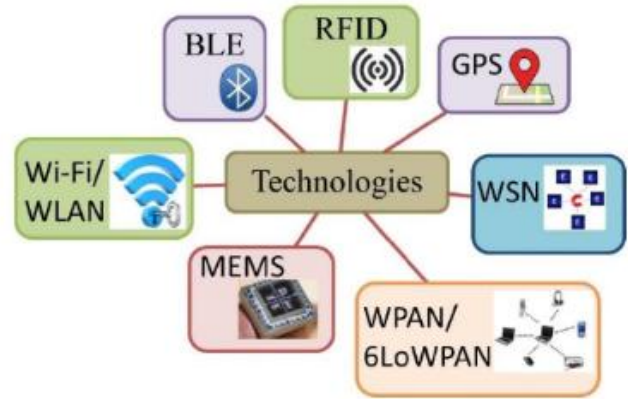

Figure 4 : Deploy Smart Healthcare technologies [18]

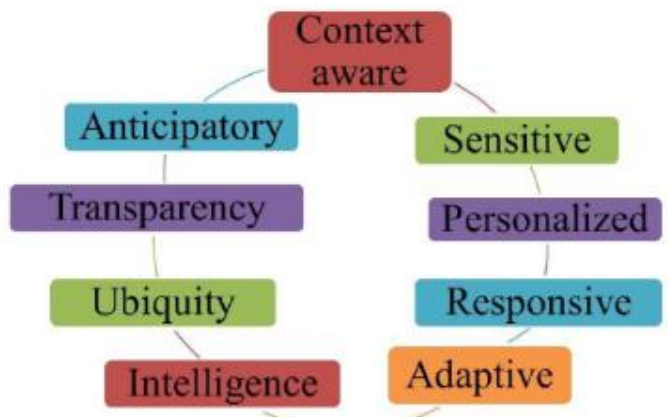

Figure 5: Smart Healthcare characteristics [18] 
A healthcare architecture includes several libraries and environments. The platform of healthcare classified in three main platforms:

1. computing platforms

2. service platforms

3. network platforms

To interconnection of different architectures the network platforms consider as libraries of networking.

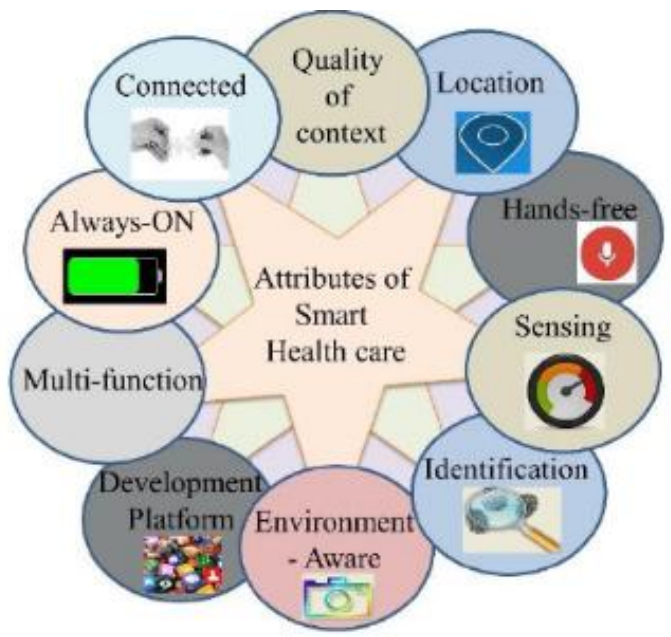

Figure 6: Smart Healthcare attributes [18]

\section{SMART HEALTH RECORDS}

In digital format when patient data are stored electronically then this is known as smart health record [16]. These smart records can access by several other health care systems. These records can be shared through other information networks and exchanges, enterprise-wide information system and network-connection. These records have wide data of medical history, radiology images, personal statistics like weight, billing information and age, demographics, medication and allergies, immunization status, laboratory test results etc [17].

For increasing the quality of care of patients the smart health records are developed. Through the care management programs the provider use data from records of patients. For improving the effectiveness and nature of human services the associated health records empower self-care benefits and can work with remote access. The record of smart healthcare give a total arrangement of work self-sufficiently; when it require an input arrangements from the clinicians it can straightforwardly associated with them. For individuals or medical clinics dependent on cost, design and power the medicinal services system can be created.

\section{SECURITY, PRIVACY AND CONFIDENTIALITY}

It is very necessary to provide a secure patient record in health care sector. Regardless, institutions of health move in the direction of improved access. In 2013 Neame defined that they have a commitment to guarantee that moral, security and privacy guidelines should complete. There ought to be a balance among access of data and protection of that data, because patient data is private.

It is very necessary for healthcare institutions to the secrecy and trustworthiness of patient data. it is currently a legitimate prerequisite. In any case, in smart health records it is very difficult. The privacy of record of Patient is very important because in many cases like HIV status on account of shrewd consideration could bring about social shame, forswearing of health advantages and loss of business. Moreover if it provides unapproved access to billing data then it can be the cause of financial losses for patients.

Table $1:$ Smart care meets security issues

\begin{tabular}{|l|l|l|}
\hline $\begin{array}{l}\text { Area of security } \\
\text { concern }\end{array}$ & Example of category & $\begin{array}{l}\text { How Smart-care meets the security } \\
\text { need }\end{array}$ \\
\hline Physical safeguards & $\begin{array}{l}\text { Screening rooms and other offices where } \\
\text { computer software is kept }\end{array}$ & $\begin{array}{l}\text { All offices and screening rooms are } \\
\text { lockable }\end{array}$ \\
\hline $\begin{array}{l}\text { Administrative } \\
\text { safeguards }\end{array}$ & $\begin{array}{l}\text { Preventing misuse of patient information } \\
\text { by authorised user }\end{array}$ & Staff training and monthly user auditing \\
\hline Technical safeguards & $\begin{array}{l}\text { Unauthorised access (privacy and } \\
\text { confidentiality) }\end{array}$ & $\begin{array}{l}\text { Staff access passwords } \\
\text { Automatic account logoff after inactivity }\end{array}$ \\
\cline { 2 - 3 } & $\begin{array}{l}\text { Those whole steal patient identity and } \\
\text { their smartcards (privacy and } \\
\text { confidentiality) }\end{array}$ & Patient access pin numbers \\
\cline { 2 - 3 } & Back up and device disposal & $\begin{array}{l}\text { Standard device disposal protocols } \\
\text { available and all data is backed up }\end{array}$ \\
\cline { 2 - 3 } & Backup and duplication & $\begin{array}{l}\text { Access to backup and duplication } \\
\text { restricted to senior staff members only }\end{array}$ \\
\cline { 2 - 3 } & $\begin{array}{l}\text { Hackers or large security breach } \\
\text { (Firewalls and transmission modes) }\end{array}$ & $\begin{array}{l}\text { No transmission mode } \\
\text { Uses standard anti viruses that are not } \\
\text { recommended for this purpose }\end{array}$ \\
\hline $\begin{array}{l}\text { Policies and } \\
\text { procedures }\end{array}$ & Access procedures & User protocols in place \\
\hline $\begin{array}{l}\text { Organisational } \\
\text { requirements }\end{array}$ & Notification for breach & Breach notification protocols in place \\
\hline
\end{tabular}

\section{AN APP PLATFORM FOR HEALTHCARE}

SMART Health IT is an application that provides standards based technology platform that empowers innovators and over the healthcare system it run securely and consistently.
To enhance research, public health and clinical care by using data warehouse or smart health records the application support SMART human services experts, standard, doctors and patients. 


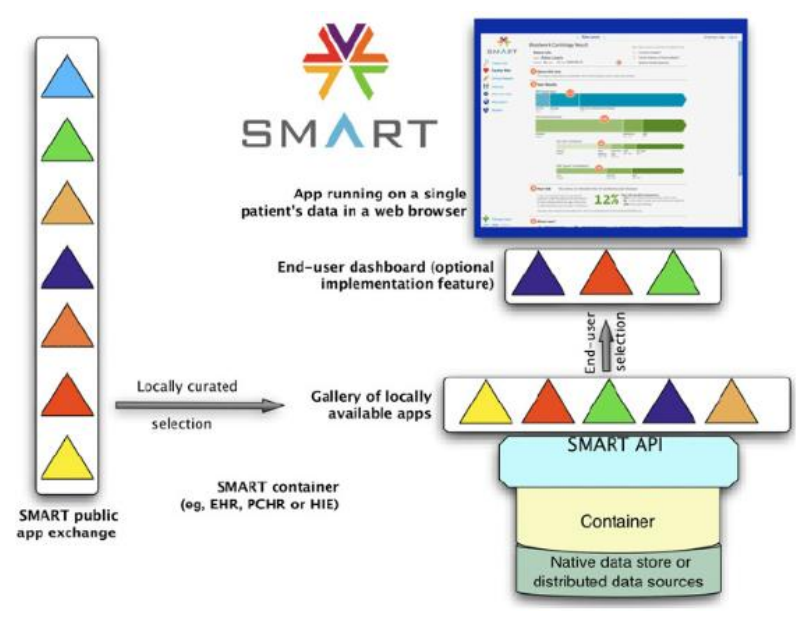

Figure 7: smart app

\section{Meaning of Smart}

By using Smart word for application of healthcare it provide several advantages to healthcare institutions, app developers, patients, and public health and healthcare providers.

Developer of Application - when it is connected with smart health record of customer it reduce the complexity and cost. It can use open source resources and tools.

Healthcare Providers and Patients- the new health provider introduce new data and capabilities to previous existing system and combined the data that are get from the clinical records from devices, patient reports and information from sensors.

Institutions based on Healthcare- on a library of innovate apps by streamlining internal smart health records drawing and customization projects, improve return on return on investment.

Public Health - the application combined provide workflow, functionality and ideas.

\section{CLOUD-BASED SMART HEALTH MONITORING}

In patient wellbeing checking devices the present advancements are based on cloud. the networks of cloud enables restorative experts to be told as suitable, empowers information stockpiling and examination and gives generous healthcare computing features [19]. Because of the expanding utilization of IoT-empowered smart devices, Appropriation of the cloud in social insurance will proceed to advance and quicken. For analytical power the interpreting healthcare data the Watson Health offer cloud-based access [20].

\section{CONCLUSION}

On the off chance that there is no health record history of a patient, at that point it will be exceptionally hard for treating in various emergency clinics. Particularly for voyagers and on account of crisis it can destroy the propitious treatment and determination. On the off chance that the shrewd wellbeing records depend on Cloud, at that point there is higher challenges for insurance and security, the open troubles of interoperability and joining and furthermore absence of consistently support for the high availability of prosperity history. The savvy wellbeing records that are now exists can store just restricted about of information of patients and they put away information just for explicit emergency clinic and don't bolster flexibility of patients transversely over different restorative facilities. The innovation that utilization better treatment for patients, improve personal satisfaction for everybody and give better indicative devices can be consider as smart health.

\section{REFERENCES}

1. Divyashikha Sethia Daya Gupta, Huzur Saran, "Smart health record management with secure NFC-enabled mobile devices", Smart Health, 22 November 2018.

2. Lasi H, Fettke P, Feld T, Hoffmann M. Industry 4.0. Bus Inf Syst Eng. 2014;6:239-42.

3. Lu H, Lv S, Jiao X, Wang X, Liu J. Maximizing information diffusion in the cyber-physical integrated network. Sensors (Basel) 2015;15:28513-30.

4. Schoenberger CR. The Internet of things. Forbes. 2002;169:155-60.

5. Hossain MS, Muhammad G. Cloud-assisted industrial Internet of things (IIoT)-enabled framework for health monitoring. Computer Netw. 2016;101:192-202.

6. Wang HR, Ding J, Xie W, Zhang D, Wang Y, Li YF. Research on medical-health cloud computing technology based on big data. Basic Clin Pharmaco Toxicol. 2016;118:46.

7. Kalid N, Zaidan AA, Zaidan BB, Salman OH, Hashim M, Muzammil H. Based real time remote health monitoring systems: a review on patients prioritization and related "big data" using body ensors information and communication technology. J Med Syst. 2017;42:30.

8. Steinhubl SR, Muse ED, Topol EJ. The emerging field of mobile health. Sci Transl Med. 2015;7:283rv3.

9. Harrison V, Proudfoot J, Wee PP, Parker G, Pavlovic DH Manicavasagar V. Mobile mental health: review of the emerging field and proof of concept study. J Ment Health. 2011;20:509-24

10. Oh SY, Chung K, Han JS. Towards ubiquitous health with convergence. Technol Health Care. 2016;24:411-3.

11. Eidam S, Redenz A, Sonius D, vom Stein N. Ubiquitous healthcare - Do the health and information technology sectors converge? Int J Innov Technol Manag.

12. Jayaram NM, Khariton Y, Krumholz HM, Chaudhry SI, Mattera J, Tang F, et al. Impact of telemonitoring on health status. Circ Cardiovasc Qual $\begin{array}{llll}\text { Outcomes. } & 2017 & \text { Dec;10(12) doi: }\end{array}$ 10.1161/CIRCOUTCOMES.117.004148. pii: e004148.

13. Moulin T, Simon P. E-Health - the internet of things and telemedicine. Corresp MHND. 2016;20:58-64.

14. Giansanti D. Introduction of medical apps in telemedicine and ehealth problems and opportunities. Telemed J E Health. 2017;23:773-6.

15. El-Miedany Y. Telehealth and telemedicine: how the digital era is changing standard health care. Smart Homecare Technol Telehealth. 2017;4:43-51.

16. Gunter TD, Terry NP (March 2005). "The emergence of national electronic health record architectures in the United States and Australia: models, costs, and questions". Journal of Medical Internet Research. 7 (1): e3. doi:10.2196/jmir.7.1.e3. PMC 1550638. PMID 15829475.

17. "Mobile Tech Contributions to Healthcare and Patient Experience". Top Mobile Trends. 22 May 2014. Archived from the original on 30 May 2014. Retrieved 29 May 2014.

18. Sundaravadivel, Prabha \& Kougianos, Elias \& Mohanty, Saraju \& Ganapathiraju, Madhavi. (2018). Everything You Wanted to Know about Smart Health Care: Evaluating the Different Technologies and Components of the Internet of Things for Better Health. IEEE Consumer Electronics Magazine. 7. 18-28. 10.1109/MCE.2017.2755378.

19. Xu BY, Xu L, Cai H, Jiang L, Luo Y, Gu Y. The design of an m-Health monitoring system based on a cloud computing platform. Enterp Inf Syst. 2017;11:17-36

20. IBM . Amonk (NY): IBM; 2018. Jan, Watson health: get the facts [Internet] [cited 2018 Jul 17]. 